\title{
Review of Brush Painting for Cost-efficient Paintable Electronics
}

\author{
Received 11 January, 2021; accepted 28 January, 2021
}

\author{
Gye-Won Kim ${ }^{a}$, Yong Jun Kim ${ }^{b}$, and Han-Ki Kim ${ }^{\mathrm{b}, *}$ \\ a Department of Fine Arts, Sungkyunkwan University, Seoul 03063, Republic of Korea \\ bschool of Advanced Materials Science and Engineering, Sungkyunkwan University, Suwon 16419, Republic of Korea
}

*Corresponding author E-mail: hankikim@skku.edu

\begin{abstract}
We briefly review a recent research development of brush-painting technologies for cost-efficient paintable electronics. Although several techniques of solution-coating, such as slot-die coating, inkjet printing and screen printing, have been developed for printable electronics, there have been great advances in brush-painting technologies and paintable electronics. This review demonstrates distinctive characteristics of brushpainting process and its applications, including paintable interconnectors, paintable transparent electrodes, and paintable semiconductors. In particular, we focus on the characteristics of brush paintable conducting polymer, metal nanowire and nanoparticles for paintable electrodes. In addition, we discuss the current status and issues of brush painting-based paintable electronics. Furthermore, we introduce promising examples of visual art works based on brush-paintable electronics. By comparing typical solution coating methods with brush-painting method, we suggest potentials of brush-painting electronics for the next generation technologies.
\end{abstract}

Keywords: Brush painting, Paintable electronics, Interconnectors, Electrodes,Visual art works

\section{Introduction}

The rapid advancement of solution-based printing technologies has created cost-effective printing electronics, including printable displays, printable photovoltaics, and printable thin film transistors (TFTs) [1-3]. Various coating methods have been suggested for solution-based printing technologies, due to their simple and atmospheric process, such as inkjet printing, screen printing, spray, gravure printing, and flexography printing. However, these types of printing technologies require a large amount of solution for coating test and result in the increase of the total cost. In this regard, brush-painting technologies have attracted great attention due to its process simplicity and usage of a small amount of ink. The method of brush-painting can compete with other printing techniques in the area of printing electronics because it has the following advantages: simple control of film thickness from $\sim 10 \mathrm{~nm}$ to a few hundred nanometers (due to fast solidification of films on a hot-substrate); shear stress-induced ordering of polymer chains or 1-dimensional nanostructures caused by the contact with brush; and processability on various flat and textured substrates [4]. In particular, brush-painting technologies could be easily incorporated into traditional Korean paper (Hanji), both of which combination could successfully realize paper electronics. For these reasons, brush-painting methods have been employed in academic research to test a small amount of functional ink for printable or paintable electronics on flexible substrate or paper [5]. Recently, many research papers have reported the possibility of ultra-low-cost brush-painting technologies in fabrication of organic solar cells, organic TFTs, sensors, transparent conducting electrode (TCE), radiofrequency identification (RFID), wearable devices, and interconnectors, as shown in Fig. 1 [4-14]. To realize paintable electronics, the materials of semiconductors, insulators, and electrode should be directly patterned by a simple process of brush painting. In particular, cost-effective paintable electronic devices could be commonly fabri-

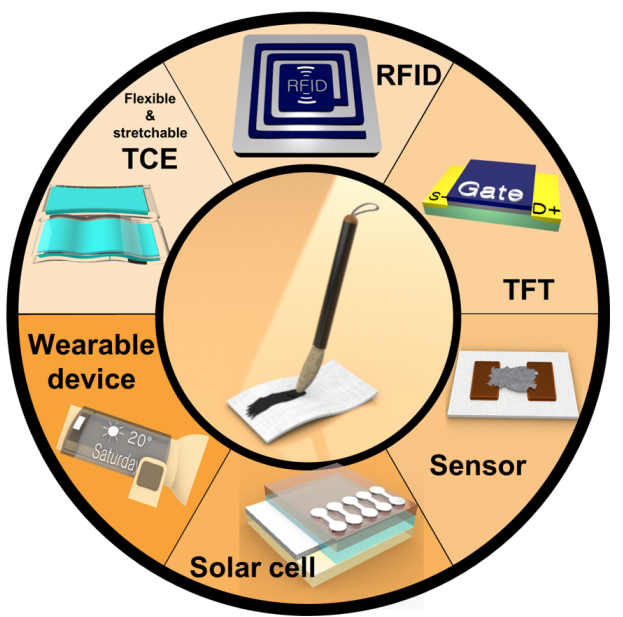

Figure 1. Applications of brush-painting technologies. Clockwise from the top these are RFID, TFT, Sensor, Solar cell, Wearable device, and TCE, respectively.

cated by using a normal paintbrush. Figure 1 illustrates several cases of the applications of brush painting, benefited from its ultra-low cost in coating process. Nevertheless, there is no review paper that widely covers the actual advantages and achievements of brush painting technologies.

In this review, we report on recent developments in brush painting technologies in three ways. First, we explain the distinctive characteristics of typical brush-painting technologies adopted for coating functional semiconductor, insulator, and metal ink. Second, we discuss in detail promising applications of brush-painting technologies in photovoltaics, TFTs, and electronics. Finally, we demonstrate the potential of brush painting for next-generation coating technologies. From this 


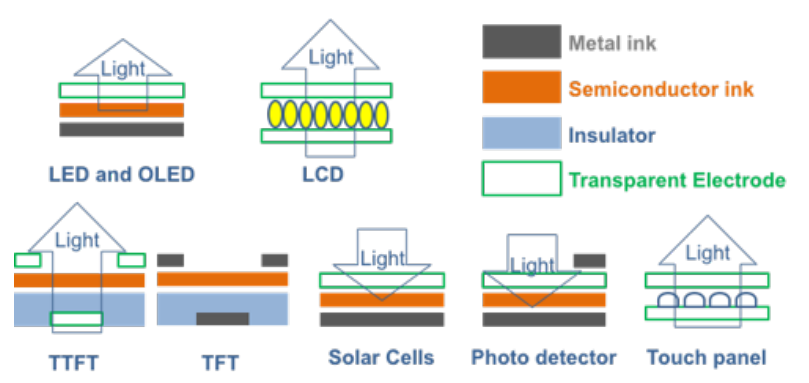

Figure 2. Schematic device structure which fabricated by brush painting technologies.

review, readers will be able to understand the recent advancement of brush-painting technologies.

\subsection{Characteristics of brush painting technolo- gies}

To fabricate printable or paintable optoelectronic devices, we first prepared functional ink such as semiconductor, insulator, and conductive electrode inks. As shown in Fig. 2, most of optoelectronic devices could be fabricated by the combination of functional metal, semiconductor, insulator, and transparent electrode ink. Therefore, multicoating of functional inks by a paintbrush could realize the typical optoelectronic devices like vacuum-based coating process. In particular, patterned electrode materials such as metal and transparent electrode are a key component for printable and paintable optoelectronic devices. Because the electrode materials could be simply patterned by direct brush painting among several components, most brush painting has been employed in direct patterning of electrode on devices $[4,5,12]$.
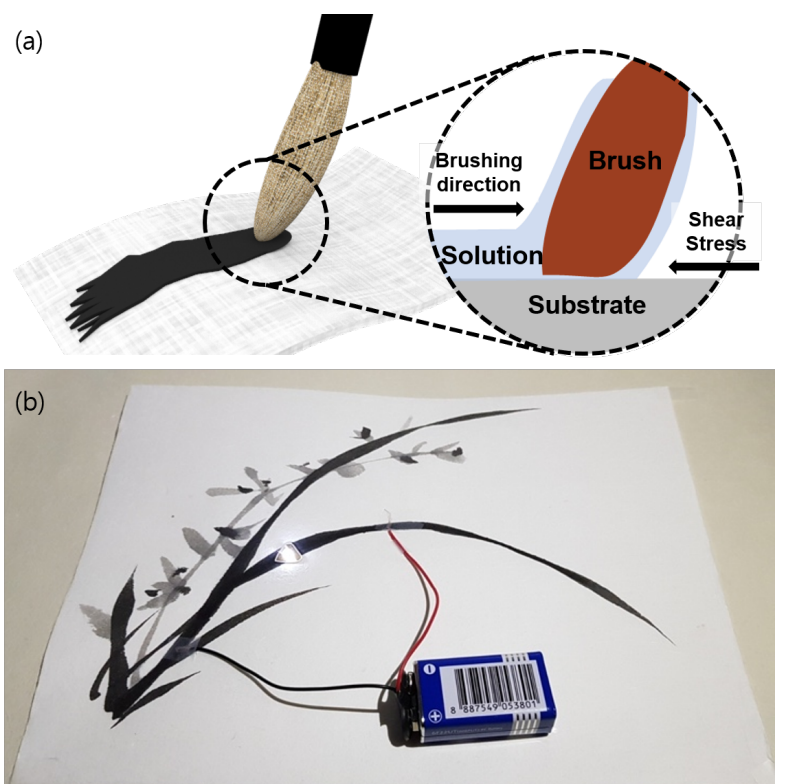

Figure 3. (a) Schematic of brush-painting process for coating and (b) brushpainted picture using black conductive ink for realizing brush paintable inter connector.

Figure 3(a) shows a schematic of the brush-painting process using a narrow paintbrush normally used for writing and painting. According to its size and shape, various types of paintbrush could be employed in different pattering processes of electrodes. In general, flat and small paintbrushes made of nylon fibrils were used to fabricate paintable and writable devices $[4,5,12]$. In the process of paint-

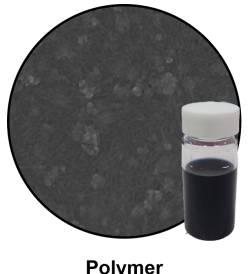

Polymer

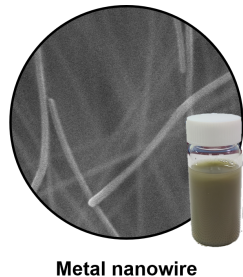

Metal nanowire

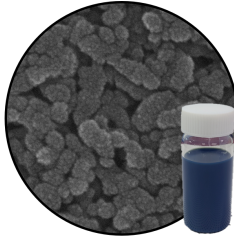

Nano Particle
Figure 4. The variable solution appliable to brush painting. The right ink is representative material. From left, these are poly(3,4-ethilenethiophene) polystyrenesulfonate, Ag nanowire solution, and ITO nanoparticle.

ing, the shear stress of paintbrush led to the lateral alignment of ink and the formation of film on substrate. The movement of the bristles is guided by their moment of inertia, the repulsive force from the substrate, and the external force on the brush applied by a person who holds it. The bristles absorbed functional ink and left a trace on the rigid or flexible substrate, as shown in Fig. 3(a). In our previous work, we introduced this brush-painting method for coating Ag nanowire(NW) and PEDOT:PSS mixed ink [4]. As we explained, the shear stress of the bristles resulted in a uniformly coated conducting PEDOT:PSS layer, which acts as a conducting matrix for the Ag NW network. Therefore, we fabricated fairly thin paintable electrodes on polyurethane (PU) substrates because the bristle of the paintbrush directly contacted the PU substrate, unlike conventional bar-coating or slot die coating. The combination of the shear stress of the bristles and the friction force on the PU substrate led to very thin brush-painted Ag NW/PEDOT:PSS electrodes. Recently we also reported the application of brush-painting technology on traditional Korean paper named Hanji, using black conductive ink [5]. As shown in Fig. 3(b), the brush-painted interconnector with black conductive ink was realized on typical Hanji paper using a nylon fibril paintbrush with a width of $1.5 \mathrm{~cm}$. Owing to the low sheet resistance $(11.7 \mathrm{Ohm} / \mathrm{square})$ of the brush-painted picture, the serially connected white light emitting diode (LED) was turned on when DC power was applied to the brush painted picture. This demonstrates the feasibility of brush-painting technology for drawing interconnectors on substrate or paper.

For the purpose of making paintable electronic devices, we must design and synthesize the functional ink apt for brush painting. By mixing water and functional solute like polymer, metal nanowire, and oxide nanoparticles, we could simply synthesize ink for brush painting, as illustrated in Fig. 4. For example, Ag nanowire and PEDOT:PSS mixed ink could be used for brush painting of interconnectors. Ag NWs (length $25 \mu \mathrm{m}$, width $30 \mathrm{~nm}$, DI water $0.1 \%$ ) and conductive PEDOT:PSS (Clevios HTL Solar) mixed ink ( $20 \mathrm{~mL}: 1 \mathrm{~mL})$ were mixed for Ag NW/PEDOT:PSS hybrid electrodes on stretchable PU [4]. By combining different types of functional inks, as shown in Fig. 4, we can easily paint electronic devices on flexible substrate or papers.

\subsection{Applications of brush painting technologies}

A simple process of brush painting can be applied to fabricate a single layer or a full layer of devices. As shown in Fig. 5, transparent electrode for organic solar cells could be fabricated by the method of brush painting. Figure 5(a) shows the coating sequence of hybrid transparent electrode for flexible organic solar cells and real image [5]. Seo et al. reported that Ti-doped $\mathrm{In}_{2} \mathrm{O}_{3}$ (TIO)/Ag NW/TIO electrodes with brush-painted Ag NW interlayer. The insertion of brush-painted Ag NW between TIO layer led to the improvement of electrical and mechanical properties of hybrid electrodes. The optimized TIO/Ag NW/TIO electrode with brush-painted Ag NW has a low sheet resistance of $9.0 \mathrm{Ohm} / \mathrm{square}$ and high optical transmittance of 85.14 $\%$. In addition, the $\mathrm{TIO} / \mathrm{Ag} \mathrm{NW} / \mathrm{TIO}$ electrode led to the improvement of mechanical properties due to the brush-painted Ag NW network inserted in it. Therefore, the high efficiency of the organic solar 


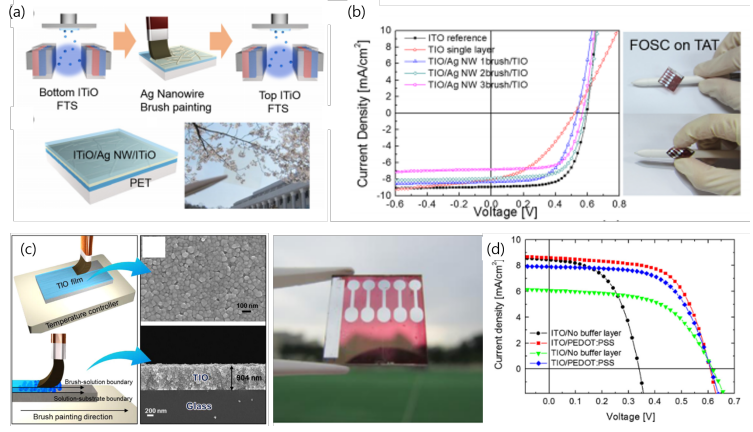

Figure 5. (a) The schematic of brush-painted flexible TIO/Ag NW/TIO electrode for organic solar cells fabrication and (b) J-V curve [14], (c) 2014, AIP Publishing LLC. (c) Fabrication method of brush-painted TIO electrode for organic solar cells, FESEM surface, cross section image, real image, and J-V curve of brush painted solar cell [6] ๔ 2013, Elsevier.
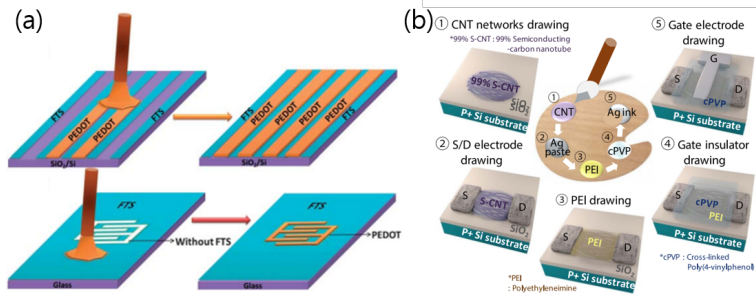

Figure 6. (a) (Reprinted (Adapted) from (Qi et al., J. Mater. Chem. C, 1, 3072 (2013)), (c) The Royal Society of Chemistry 2020) and (b) (Reprinted (Adapted) from (Park et al., Nanotechnology, 31, 32 LT01 (2020)), (c) 2020 IOP Publishing Ltd) is a fabrication method image of brush-painted organic thin film transistor electrode.

cells could be realized on the hybrid transparent electrodes, as shown in Fig. 5(b). It showed that the power conversion efficiency $(3.007$ $\%)$ of the organic solar cells with hybrid electrodes is better than that $(1.645 \%)$ of the organic solar cells with TIO single layer. Figure 5(c) showed a brush-painted TIO electrode and application in organic solar cells [6]. Using TIO nanoparticle ink, Jeong et al., reported simple brush-painted TIO electrodes for organic solar cells. Brush-painted TIO nanoparticle films were proven having uniform surface morphology by FE-SEM image. The brush-painted TIO nanoparticle electrode showed a low sheet resistance of $28.55 \mathrm{Ohm} /$ square and high optical transmittance of $85.48 \%$. The organic solar cells fabricated on the brush-painted TIO nanoparticle electrode showed a power conversion efficiency of $2.94 \%$ indicating that brush-painted TIO nanoparticles films is a promising cost-effective transparent electrode for organic solar cells. As shown in Fig. 5, the method of brush painting could be applied as coating process for transparent electrode in fabrication of cost-efficient organic solar cells.

Full brush-painted devices were suggested by Qi et al. and Park et al. as shown in Fig. $6[8,9]$. Figure 6 shows a schematic fabrication process of organic TFTs with all over brush painting. As shown in Fig. 6(a), top gate organic TFT with all over brush painting was realized by using hydrophobic $1 \mathrm{H}, 1 \mathrm{H}, 2 \mathrm{H}, 2 \mathrm{H}$-perfluorooctyltriethoxysilane (FTS) self-assembled monolayer patterning. All components of organic TFTs such as PEDOT:PSS (source and drain), P3HT or PBTTT (active layer), PMMA (insulative layer), and Ag (gate layer) were realized by the process of brush painting (without FTS layer). P3HT and PBTTT based top gate organic TFT with all over brush painting shows maximum mobilities of 0.011 and $0.14 \mathrm{~cm}^{2} \mathrm{~V}^{-1} \mathrm{~S}^{-1}$, respectively. Another type of organic TFTs with all over brush painting was also suggested as shown in Fig. 6(b). Semiconducting carbon nanotube (CNT), Ag pasteand ink, and cross-linked poly (4-vinylphenol) (cPVP) were used as an active layer, source-drain and gate, and insulating layer, respectively. Also, polyethyleneimine (PEI) can easily convert P-type CNT into N-type CNT. Therefore, the PEI treat-

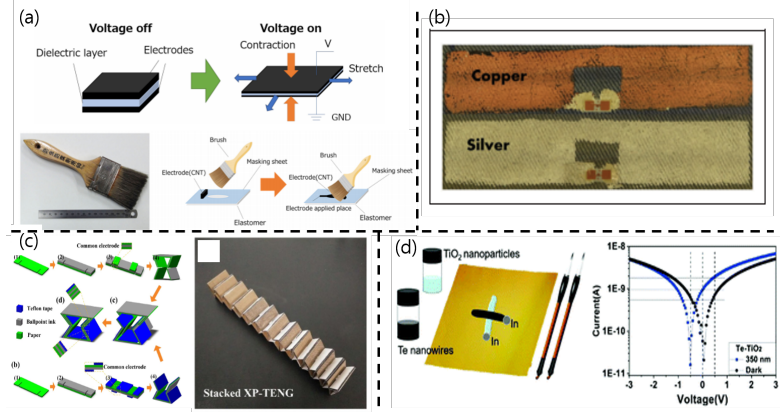

Figure 7. Various application of brush painting process. Brush painted (a) actuator [16] ( 2020, MDPI, (b) RFID [10] (c) Copyright 2020 by SAGE publication), (c) generator [15] ( 2020 Elsevier, and (d) photodetector [11] @2020, The Royal Society of Chemistry.

ment using brush-painting process made it possible to achieve cost efficiency complementary inverters. The brush-painted $\mathrm{P}$ - and $\mathrm{N}$ - type CNT-TFT exhibited on/off ratio up to $10^{3}$ with a low operating voltage. These works suggested that cost effective organic TFT could be realized by adopting a simple process of brush painting.

Figure 7 shows other various applications (actuator, RFID, generator, photo detector) using a brush painting process $[10,11,15,16]$. Figure 7(a) showed brush-painted CNT powder dielectric elastomer actuator (DEA). The CNT ink could be simply pained on stretchable or dielectric elastomer as an electrode of DEA. Since a good stretchability of the CNT electrode, DEA showed a good stretchability and the radial strain of $52 \%$ [16]. In addition, DEA with the square electrode that has oriented by brush painting shows anisotropy properties. Brush painting directly executed on the fiber layer became a powerful means of making wearable electronic devices. Figure 7(b) is textile-integrated RFIDs made by brush painting and these materials were the key components enabling wearable devices. Wang et al. reported that copper and silver ink could be painted by brush on $100 \%$ cotton fabric as antennas for RFID [10]. The optimal copper and silver tag patterned on cotton substrate generated by brush painting showed maximum read ranges of 6 and $10 \mathrm{~m}$. Also, for a successful application of wearable devices, Kazmi et al. applied a protective layer using regular textile glue (Gutermann Creativ HT2), epoxy (ESL 243 White epoxy coating), and silicone rubber (Sugru) brush painting to RFID [17]. They demonstrated that RFID worked well despite of 15 times washing cycle. These results indicate that the brush painting process is suitable for a wearable application. As a simple energy harvesting device, novel X-shaped paper triboelectric nanogenerators (XP-TENGs) was suggested by Xia et al. as shown in Fig. 7(c) [15]. As a conductive electrode, the ink extracted from ball point pen was painted on paper by brush. The XP-TENGs showed maximum power generation values of $326 \mathrm{~V}, 45$ $\mu \mathrm{A}$, and $542.22 \mu \mathrm{W} / \mathrm{cm}^{2}$. In addition, they had proposed a stacked XP-TENGs as shown in right of Fig. 7(c), with the aim of enhancing the power generation values. Finally, the stacked XP-TENGs successfully light up high-power blue LEDs. The type of solution that can be used in painting process usually have advantage of high aspect ratio and broad surface. Therefore, brush painting is an effective method for heterojunction device. As heterojunctions devices, Zhang et al. suggested $\mathrm{Te} / \mathrm{TiO}_{2}$ and $\mathrm{Te} / \mathrm{ZnO} \mathrm{UV}$ photodetector using brush painting method as shown in Fig. 7(d) [11]. They simply wrote $\mathrm{TiO}_{2}$ or $\mathrm{ZnO}$ nanoparticles on commercial paper and then, as the rewriting Te nanowires overlapping with $\mathrm{TiO}_{2}$. As a result, both $\mathrm{Te} / \mathrm{TiO} \mathrm{O}_{2}$ and $\mathrm{Te} / \mathrm{ZnO}$ UV photoreactors effectively generated photocurrent about 1 $\mathrm{nA}$ at $0 \mathrm{~V}$ with wave of $350 \mathrm{~nm}$. Table 1 summarized performance of various devices fabricated by brush painting process. Brush painting can be adopted as an effective method throughout electric devices, and if further research is proceeded, it will be an important key to realize future-oriented, low-cost, high-efficiency and eco-friend coating process. 
Table 1. Comprehensive properties of brush painted various applications.

\begin{tabular}{|c|c|c|c|c|}
\hline Application & Material & Electrical property & Optical property & Mechanical property \\
\hline Stretchable electrode & Ag NW and PEDOT:PSS & 19.7 Ohm/square & $88.64 \%$ & $30 \%$ strain \\
\hline Paper electrode & $\begin{array}{l}\text { Ag NW, PEDOT:PSS, and } \\
\text { Muk }\end{array}$ & $11.7 \mathrm{Ohm} /$ square & - & $\begin{array}{l}1 \mathrm{~mm} \text { bending radius } \\
\text { (both inner and outer) }\end{array}$ \\
\hline Organic solar cells & Ag NW & $9.01 \mathrm{Ohm} /$ square & $85.14 \%$ & $\begin{array}{c}5 \mathrm{~mm} \text { and } 6.5 \mathrm{~mm} \text { bending } \\
\text { radius } \\
\text { (inner and outer) }\end{array}$ \\
\hline Organic solar cells & TiO nanoparticle & $28.25 \mathrm{Ohm} / \mathrm{square}$ & $85.48 \%$ & Rigid substrate \\
\hline TFT & $\begin{array}{l}\text { PEDOT:PSS treated by } \\
\text { dimethyl sulfoxide } \\
\text { (source and drain) }\end{array}$ & $50 \mathrm{~S} / \mathrm{cm}$ & - & - \\
\hline Actuator & CNT & $\begin{array}{l}2.58 \mathrm{MOhm} \\
\text { (perpendicular) } \\
5.56 \mathrm{MOhm} \\
\text { (parallel) }\end{array}$ & - & stretchable \\
\hline RFID & Cooper and Silver & $0.6 \mathrm{Ohm} \sim 31.2 \mathrm{Ohm}$ & - & - \\
\hline Generator & Ball point pen ink & $6 \mathrm{kOhm} /$ square & - & - \\
\hline
\end{tabular}

\subsection{Applications of brush painting in arts (Prof. G-W Kim)}

Brush painting with functional ink can provide artists innovative modes of expression and communication due to the three following reasons. First, the Ag nanowire-based ink (BCI) can broaden the limited material options given to traditional East Asian paintings. In general, the three main materials of traditional East Asian paintings consist of paper, brush, and sumi ink. Once the BCI is adopted as a new material of painting, the lines and forms that an artist executes can be easily transformed into highly conductive electrodes. As illustrated in Fig. 8(a), the stem of plum tree, one of the most iconic subject-matters of traditional ink painting, can provide an electronic matrix on which the blue embedded LEDs can be illuminated. The seemingly plainlooking plum tree can be dramatically turned into a specific type of plum tree that has a glowing and dazzling effect due to electronic light. Second, the BCI ink can be mixed with oil-based pigments as well, and accordingly it can be adopted as a new material for Western oil painting. Figure $8(\mathrm{~b})$ is a contemporary abstract painting that the artist draws by means of a normal brush and the mixture of the BCI and oil pigments. This is an all-over painting that simply contains interconnected lines and forms. This modern style painting, however, becomes a contemporary electronic art, once the colorful LEDs, attached onto the connected lines, turn on and create unexpected illuminating effects. By using a simple means of brush and the BCI, the artist can provide viewers a new experience of painting with emitting colorful light. Third, artist can adopt Ag NW and PEDOT:PSS mixed ink as an effective material for contemporary media-installation art. Figure 8(c)
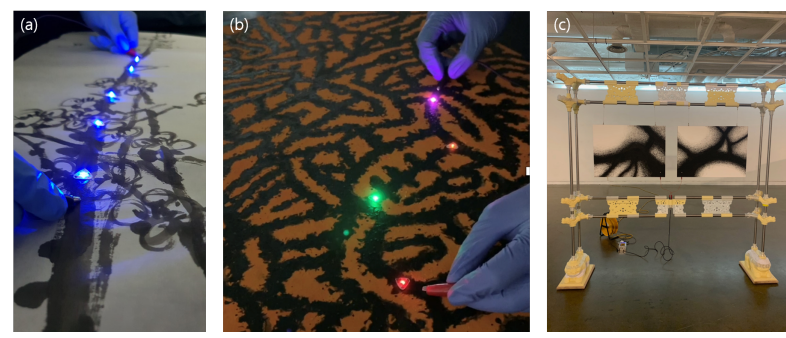

Figure 8. (a) Brush painting of a plum tree on a Hanji substrate, where blue LEDs are embedded in the form of a serial connection. Once DC power was applied to the LEDs attached onto the stem of the plum tree, all of the LEDs were turned on simultaneously. (b) Brush painting of abstract lines on a canvas. The black lines formed by brush painting act as an interconnector for illuminating the LEDs. (c) Brush painted antenna formed by Ag NW and PEDOT:PSS mixed ink. shows a mixed-media installation work that consists of two panels of black ink paintings at the center and 3D printed scriptures surrounding them. The two paintings are connected into a speaker at the bottom, and when DC power is applied, those paintings can be functioned as an antenna to transmit the received sound sources into the speaker. As a consequence, viewers can look at visual art works, while listening to the transmitted sounds simultaneously. In other words, a simple mixture of Ag NW and PEDOT:PSS can open up a new possibility of multi-sensory expression, which has been consistently explored in the field of contemporary mixed-media art.

\section{Conclusions}

This review reports the recent research development of brushpainting technology for simple and cost-effective solution coating process. In particular, this review has reported the feasibility of brush painting technology that enables a simple, flexible and humanoriented coating process. First, we have briefly outlined the distinctive characteristics of typical brush painting technologies for coating functional semiconductor, insulator, and metal ink. Second, we have discussed in details several promising applications of brush painting technologies in photovoltaics, TFTs, and wearable electronics. Finally, we have introduced the examples of fine art works made possible through brush painting with functional ink. Although there is extensive research on brush-paintable devices, we still found issues to be solved regarding brush painting such as difficulty in thickness control, limitation of functional ink, and difficulty of automation. However, a rapid advancement of solution-based printing electronics will provide solutions for those issues. This review will allow readers to be able to understand the current status of brush painting technology and its potentials as solution-coating process for next generation paintable electronics.

\section{Acknowledgements}

This work was supported by the Korea Basic Science Institute (KBSI) National Research Facilities \& Equipment Center (NFEC) grant funded by the Korea government (Ministry of Education) (No. 2019R1A6C1010031). 


\section{References}

[1] Z. Liu et al., Light Sci. Appl. 9, 83 (2020).

[2] L. Marasamy, A. D. R. Chettiar, F. Moure-Flores, and V. Subramaniam, Int. J. Energy Res. 19 (2020).

[3] V. Acharya, A. Sharma, N. K. Chourasia, and B. N. Pal, Emergent Mater 3, 62 (2020).

[4] J. E. Lim, S. M. Lee, S. S. Kim, T. W. Kim, H. W. Koo, and H. K. Kim, Sci Rep 7, 1 (2017).

[5] Y. J. Kim, S. Yoon, Y. H. Cho, G. Kim, and H. K. Kim, RSC Adv. $10,24631(2020)$.

[6] J. A Jeong, Y. J. Jeon, S. S. Kim, B. K. Kim, K. B. Chung, and H. K. Kim, Sol. Energy Mater Sol. Cells 122, 241 (2014).

[7] F. J. Lin, C. G, W. T. Chung, C. L. Wang, Q. Wang, H. Liu, C. S. Hsu, and L. Jiang, Adv. Mater. 29, 1606987 (2017).

[8] Z. Qi, F. Zhang, C. A. Di, J. Wang, and D. Zhu, J. Mater. Chem. C 18, 3072 (2013).

[9] J. Park, Y. Lee, B. Choi, J. Yoon, Y. Kim, H. J. Kim, M. H. Kang, D. H. Kim, D. M. Kim, and S. J. Choi, Nanotechnology 31, 32LT01
(2020).

[10] J. Wang, J. Liu, J. Virkki, T. Björninen, L. Sydänheimo, L. Cheng, and L. Ukkonen, Text. Res. J 86, 1616 (2015).

[11] Y. Zhang, W. Xu, X. Xu, W. Yang, S. Li, J. Chen, and X. Fang, Nanoscale Horiz 4, 452 (2019).

[12] J. A. Jeong, H. K. Kim, and J. Kim, Sol. Energy Mater Sol. Cells 125,113 (2014)

[13] S. S. Kim, S. I. Na, S. J. Kang, and D. Y. Kim, Sol. Energy Mater Sol. Cells 94, 171 (2010).

[14] K. W. Seo, J. H. Lee, H. J. Kim, H. K. Kim, and S. I. Na, Appl. Phys. Lett. 105, 031911 (2014).

[15] K. Xia, Z. Zhu, H. Zhang, C. Du, Z. Xu, and R. Wang, Nano Energy 50, 571 (2018).

[16] H. Shigemune, S. Sugano, J. Nishitani, M. Yamauchi, N.Hosoya, S. Hashimoto, and S. Maeda, Actuator 7, 51 (2018).

[17] A. Kasmi, M. Rizwan, L. Syndänheimo, L. Ukkonen, and J. Virkki, 2016 6th Electron. Syst. Technol. Conf. ESTC 2016 (2016). 